This is the final peer-reviewed accepted manuscript of:

Gianluca Palli, Salvatore Strano, Mario Terzo, Sliding-mode observers for state and disturbance estimation in electro-hydraulic systems, Control Engineering Practice, Volume 74, 2018, Pages 58-70, ISSN 0967-0661

The final published version is available online at:

https://doi.org/10.1016/j.conengprac.2018.02.007

Rights / License:

The terms and conditions for the reuse of this version of the manuscript are specified in the publishing policy. For all terms of use and more information see the publisher's website.

This item was downloaded from IRIS Università di Bologna (https://cris.unibo.it/)

When citing, please refer to the published version. 


\title{
Sliding-Mode Observers for State and Disturbance Estimation in Electro-Hydraulic Systems
}

\author{
Gianluca Palli*,a , Salvatore Strano ${ }^{\mathrm{b}}$, Mario Terzo $^{\mathrm{b}}$ \\ ${ }^{a}$ DEI - Università di Bologna, Viale Risorgimento 2, 40132 Bologna, Italy \\ ${ }^{b}$ DII - Università degli Studi di Napoli Federico II, via Claudio 21, 80125 Napoli, Italy
}

\begin{abstract}
In this paper, the comparison between a sliding mode observer and a high-order sliding mode observer for hydraulic actuators is presented considering different sets of available measurements, parametric uncertainties and model nonlinearities. The friction acting on the hydraulic cylinder and a dead-zone in the valve have been taken into account as uncertain disturbances acting on the system. For comparison purposes, the observation results are set side by side with the ones provided by an extended Kalman filter. Several simulations have been conducted in order to analyze the performance of the estimators, particularly with respect to the possibility of estimating the disturbances and unknown dynamics acting on the system, also in presence of measurement noise. Finally, experimental results are presented to validate the effectiveness of the proposed observers in real conditions.
\end{abstract}

Key words: Hydraulic actuators, sliding-mode observers, disturbance observers, nonlinear systems.

\section{Introduction}

Over the past decade, observers have been used to improve the reliability of systems allowing fault detection and diagnostics $[1,2]$. Observers can also be adopted for reducing the number of sensors or for replacing sensors that may not be able to withstand some extreme environmental conditions [3]. Many interesting applications of observers in industrial applications are related to hydraulic actuation systems $[4,5,6,7]$. Indeed, complexity and hard working conditions of hydraulic systems, due to harsh environment and high operative load, strongly exhort to adopt state observers functional for prognostics, diagnostics and fast fault detection. Moreover, observers in hydraulic systems are a valid alternative to the installation of sensors for feedback controllers [8]. The design of state observers should take into account the nonlinearities of hydraulic actuators such as friction, pressure-flow rate relationship and dead-zone that occurs in closed center valves when the land width is greater than the port width at neutral spool position [9]. Within this context, several approaches have been proposed in literature. These methods include linear approaches, linearized model based techniques [10] and observers based on nonlinear models [11, 12]. Extended Kalman filter (EKF) has been adopted to detect faults in hydraulic actuators [13] by means of the state estimation. The approach is based on the local linearization of the nonlinear system and allows to closely track the state trajectories when compared to the linear approach of the Kalman filter [14]. Halder [15] showed a procedure to detect and isolate the sensor fault of an EHA system using conventional EKF. An unscented Kalman Filter based approach is presented in [16]. In [17], a nonlinear approach based on the State-Dependent-Riccati-Equation (SDRE) has been proposed together with experimental validation. State-based estimators based on the Kalman filter provide satisfactory results when the mathematical model of the system under consideration is known, indeed, unmodelled effects and parametric uncertainties are only managed by stochastic variables. Consequently, these approaches fail when it is not possible to model in detail the system dynamics. In addition, Kalman filter based estimators generally require long and hard calibration procedures. The high degree of parametric uncertainty that characterizes hydraulic actuators exhorts the researchers to follow robust approaches for the state estimation [18, 19]. In [20], the authors proposed a method based on robust filter structure, to compensate for the effect of the unmodelled dynamics, combined with on-line parameter adaptation, to account for the parametric uncertainty. In [21], an adaptive sliding mode observer (SMO) is proposed for the estimates of velocity and parameters of an electro-hydraulic system. Even if SMO is a valid tool for managing uncertainties, it is well known that the undesirable chattering phenomenon will degrade the estimation performance and then a low pass filter is required for the reconstruction of the uncertainties. Higher order sliding mode observers (HSMOs) can reduce chattering achieving a better sliding accuracy [22]. The use of HSMOs for state

${ }^{*}$ Corresponding author 


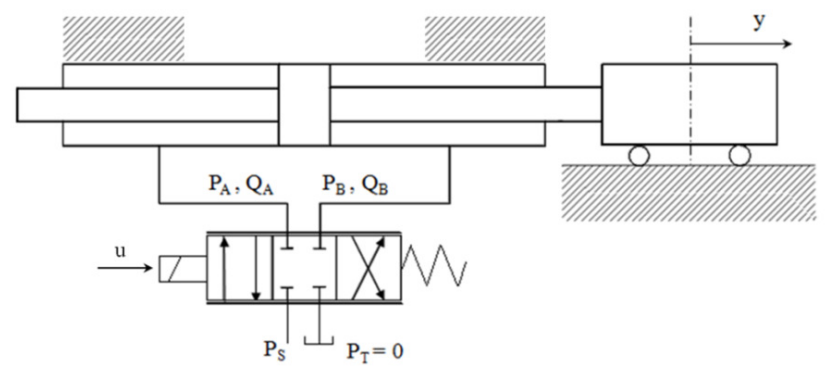

Figure 1: Schematic diagram of the hydraulic actuation system.

and unknown input estimation in uncertain nonlinear systems has been discussed in [23, 24]. In [25], the authors have presented a HSMO for the estimation of road adhesion coefficient under various surface conditions. In [26] a HSMO is proposed to estimate exactly the observable states and asymptotically the unobservable ones in MIMO nonlinear systems with unknown inputs and stable internal dynamics. In [27] a step-by-step higher order sliding mode observers for MIMO nonlinear systems with unknown inputs is described. A structural matching condition, commenting on the possibility to design such observers and to reconstruct the unknown inputs, is derived. In [28] the estimation problem of a class of SISO nonlinear Lipschitz systems with non-matching uncertainty where the distribution vector may include the unknown states is considered.

In this paper, the design of a SMO and a HSMO for hydraulic actuators is presented considering parametric uncertainties and model nonlinearities. Unlike [21], a dead-zone function has been introduced in the hydraulic actuator mathematical model in order to describe a realistic control valve. Simulations and experimental tests have been conducted in order to analyze the performance of the estimators, particularly with respect to the possibility of estimating the disturbances and unknown dynamics acting on the system, also in presence of measurement noise. This feature is particularly useful for system diagnosis and fault detection. Moreover, comparisons with the largely used EKF are illustrated.

The paper is organized as follows: a fifth-order nonlinear model of the hydraulic actuator is derived in Section 2 taking into account dead-zone, friction and the nonlinear pressure-flow rate relationship; Section 4 and 5 focus on the SMO and on the HSMO, respectively. Section 6 shows the simulation results, while Sec. 7 reports the experiments performed of the test platform. Finally, conclusions are drawn in Section 8.

\section{System Description and Modeling}

The hydraulic actuation system under consideration consists mostly of a double-rod hydraulic cylinder and a proportional valve. The hydraulic cylinder is linked to a mass that moves on a linear guide (Fig. 1). For the derivation of the mathematical model, some assumptions are made: the tank pressure $P_{T}$ is equal to zero, the fluid properties are not dependent on the temperature, the piston areas and the chamber volumes are equal, the internal and external fluid leakages are negligible. The time dependence of the variables is omitted in the equations for brevity. The dynamics of the movable mass displacement $y(t)$ is governed by:

$$
m \ddot{y}+b \dot{y}+F_{f}(\dot{y})=A_{p} P_{L}
$$

where $m$ is the mass of the load, $b$ is the viscous friction coefficient, $F_{f}(\dot{y})$ is the friction force, $A_{p}$ is the piston area, $P_{L}(t)=P_{A}(t)-P_{B}(t)$ is the load pressure, $P_{A}(t)$ and $P_{B}(t)$ are the pressures inside the two chambers of the cylinder. The friction force is represented by the following equations:

$$
F_{f}(\dot{y})= \begin{cases}F_{c} \operatorname{sign}(\dot{y})+\mu m g \operatorname{sign}(\dot{y}), & \dot{y} \neq 0 \\ Z|Z| \leq F_{c 0}+\mu_{0} m g, & \dot{y}=0\end{cases}
$$

where $F_{c}$ is the Coulomb friction force in the hydraulic actuator (assumed equal to its static value $F_{c 0}$ ), $\mu$ is the Coulomb friction coefficient of the linear guide (assumed equal to its static value $\mu_{0}$ ), $g$ is the gravitational acceleration and $Z$ is the net tangential force that acts on the actuator when it is not moving [29]. It is worth noticing that the friction model is used in simulation only for the evaluation of the observer performance. The load pressure dynamics [30] is given by:

$$
\frac{V_{0}}{2 \beta} \dot{P}_{L}=-A_{P} \dot{y}+Q_{L}
$$


where $V_{0}$ is the volume of each chamber for the centered position of the piston, $Q_{L}=\left(Q_{A}+Q_{B}\right) / 2$ is the load flow and $\beta$ is the effective Bulk modulus. The load flow depends on the supply pressure $P_{s}$, the load pressure $P_{L}$ and the valve spool position in accordance with the following equation:

$$
Q_{L}=\Psi\left(v_{e}\right) \sqrt{P_{s}-\left|P_{L}\right|}
$$

where $v_{e}(t)$ is the displacement signal of the spool valve and $\Psi\left(v_{e}\right)$ is a gain that depends on the geometry of the adopted proportional valve. The analytical expression of $\Psi\left(v_{e}\right)$ can be assumed as:

$$
\Psi\left(v_{e}\right)= \begin{cases}k_{q p}\left[v_{e}+\left(\frac{k_{q 0}}{k_{q p}}-1\right) v_{e p}\right], & v_{e}>v_{e p} \\ k_{q 0} v_{e}, & v_{e n} \leq v_{e} \leq v_{e p} \\ k_{q n}\left[v_{e}+\left(\frac{k_{q 0}}{k_{q n}}-1\right) v_{e n}\right], & v_{e}<v_{e n}\end{cases}
$$

where $v_{e n}, v_{e p}, k_{q 0}, k_{q n}, k_{q p}$ are parameters that must be identified experimentally. Equation (5) represents an asymmetric bilinear function of the valve spool position. If $k_{q 0}=k_{q p}=k_{q n}$, the function $\Psi\left(v_{e}\right)$ reduces to a linear proportional valve. If $k_{q 0}=0, \Psi\left(v_{e}\right)$ describes a dead-zone function, where $v_{e n}$ and $v_{e p}$ are the limits of the dead-zone and $k_{q n}$ and $k_{q p}$ are the gains if $v_{e}$ is negative or positive respectively. The adoption of $\Psi\left(v_{e}\right)$ is particularly efficient to describe a dead-zone function if an overlapped valve is adopted [31]. The proportional valve dynamics can be well represented by the following second order differential equation [32]:

$$
\frac{\ddot{v}_{e}}{\omega_{n v}^{2}}+\frac{2 \epsilon_{v}}{\omega_{n v}} \dot{v}_{e}+v_{e}=k_{e} u+v_{e 0}
$$

where parameters $\omega_{n v}$ and $\epsilon_{v}$ are the natural frequency and the damping ratio of the valve respectively, $v_{e 0}$ is the spool position bias, $k_{e}$ is the input gain and $u(t)$ is the valve command. Finally, the equations governing the dynamics of the whole system (movable mass + hydraulic system) are:

$$
\left\{\begin{array}{l}
m \ddot{y}+b \dot{y}+F_{f}(\dot{y})=A_{p} P_{L} \\
\frac{V_{0}}{2 \beta} \dot{P}_{L}=-A_{P} \dot{y}+Q_{L} \\
Q_{L}=\Psi\left(v_{e}\right) \sqrt{P_{s}-\left|P_{L}\right|} \\
\ddot{v}_{e}=-\omega_{n v}^{2} v_{e}-2 \epsilon_{v} \omega_{n v} \dot{v}_{e}+\omega_{n v}^{2}\left(k_{e} u+v_{e 0}\right)
\end{array}\right.
$$

The developed fifth order model fully describes the nonlinear dynamical behavior of the hydraulic actuation system and takes the nonlinear friction forces and the nonlinear flow rate distribution into account. The nonlinear system eq. (7) can be written in the following form:

$$
\left\{\begin{array}{l}
\ddot{y}=-\frac{b}{m} \dot{y}-\frac{F_{f}(\dot{y})}{m}+\frac{A_{p} P_{L}}{m} \\
\dot{P}_{L}=-\frac{2 \beta A_{P}}{V_{0}} \dot{y}+\frac{2 \beta \Psi\left(v_{e}\right) \sqrt{P_{s}-\left|P_{L}\right|}}{V_{0}} \\
\ddot{v}_{e}=-\omega_{n v}^{2} v_{e}-2 \epsilon_{v} \omega_{n v} \dot{v}_{e}+\omega_{n v}^{2}\left(k_{e} u+v_{e 0}\right)
\end{array}\right.
$$

Note that the system (8), whose state vector is given by $x(t)=\left[y(t) \dot{y}(t) P_{L}(t) v_{e}(t) \dot{v}_{e}(t)\right]^{T}$, is nonlinear in the state and affine in the input $u(t)$.

Now, the nominal system dynamics, separated from the disturbances, and the measurement equations are rewritten as:

$$
\begin{aligned}
\dot{x}(t) & =f(x(t))+g(x(t)) u(t)+\varphi(x(t), t) \\
z(t) & =h(x(t))
\end{aligned}
$$

where $z(t)$ is the output vector, $f(x(t), u(t)), \varphi(x(t), t)$ and $h(x(t))$ are the state dynamics, the disturbance and the output non-linear functions respectively, $u(t)$ is the input vector. The nominal system dynamics is then:

$$
f(x(t))=\left[\begin{array}{c}
\dot{y} \\
-\frac{b}{m} \dot{y}+\frac{A_{p} P_{L}}{m} \\
-\frac{2 \beta A_{P}}{V_{0}} \dot{y}+\frac{2 \beta k_{q} v_{e} \sqrt{P_{s}-\left|P_{L}\right|}}{V_{0}} \\
-\omega_{n v}^{2} v_{e}-2 \epsilon_{v} \omega_{n v} \dot{v}_{e}
\end{array}\right]
$$




$$
g(x(t))=\left[\begin{array}{c}
0 \\
0 \\
0 \\
0 \\
\omega_{n v}^{2} k_{e}
\end{array}\right], \quad h(x(t))=\left[\begin{array}{c}
y \\
P_{L} \\
v_{e}
\end{array}\right], \quad \varphi(x(t), t)=\left[\begin{array}{c}
0 \\
-\frac{F_{f}(\dot{y})}{m}+\eta_{y}(t) \\
\frac{2 \beta\left(\Psi\left(v_{e}\right)-k_{q} v_{e}\right) \sqrt{P_{s}-\left|P_{L}\right|}}{V_{0}}+\eta_{P_{L}}(t) \\
0 \\
\omega_{n v}^{2} v_{e 0}+\eta_{v_{e}}(t)
\end{array}\right]
$$

where $k_{q}$ is the nominal valve constant. The function $\eta(t)=\left[\eta_{y}(t), \eta_{P_{L}}(t), \eta_{v_{e}}(t)\right]^{T}$ includes unmodelled dynamics and parameter uncertainties.

\section{Extended Kalman Filter}

The derivation of the EKF is briefly recalled in the following lines, since it has been adopted for a comparative analysis. The system and the measurement eqs. (9)-(10) can be generically rewritten in a discrete-time form which also accounts for both process and measurement noise as:

$$
\begin{aligned}
x_{k} & =f_{k}\left(x_{k-1}, u_{k-1}\right)+L_{k} \phi_{k} \\
z_{k} & =h\left(x_{k-1}, u_{k-1}\right)+M_{k} g_{k}
\end{aligned}
$$

where $f_{k}(x, u)$ is the discrete-time state equation obtained by integrating eq. (9) from time $t_{k-1}$ to time $t_{k}, x_{k-1}$ and $u_{k-1}$ are the values of the state and the input vectors at the time instant $(k-1), \phi_{k}$ is the Gaussian white process noise with covariance $Q_{k}, z_{k}$ is the measurement vector at time $k$, and $g_{k}$ is the Gaussian white measurement noise with covariance $R_{k}$. The EKF is conceptually based on two fundamental steps, namely prediction (denote by the superscript ${ }^{-}$) and correction (denote by the superscript ${ }^{+}$). Denoting the generic variable estimates as $\left({ }^{\wedge}\right)$, the state prediction $\hat{x}_{k}^{-}$and the error covariance prediction $P_{k}^{-}$as well as the state correction $\hat{x}_{k}^{+}$and the error covariance correction $P_{k}^{+}$at the $k$-th time instant are given by:

$$
\begin{aligned}
\hat{x}_{k}^{-} & =f_{k}\left(\hat{x}_{k-1}^{+}, u_{k-1}\right) \\
P_{k}^{-} & =A_{k-1} P_{k-1}^{+} A_{k-1}^{T}+L_{k} Q_{k} L_{k}^{T} \\
K_{k} & =P_{k}^{-} H_{k}^{T}\left(H_{k} P_{k}^{-} H_{k}^{T}+M_{k} R_{k} M_{k}^{T}\right)^{-1} \\
\hat{x}_{k}^{+} & =\hat{x}_{k}^{-}+K_{k}\left[z_{k}-h\left(\hat{x}_{k}^{-}, u_{k}\right)\right] \\
P_{k}^{+} & =\left(I-K_{k} H_{k}\right) P_{k}^{-}
\end{aligned}
$$

with

$$
\begin{aligned}
A_{k-1} & =\left.\frac{\partial f_{k}\left(\hat{x}, u_{k-1}\right)}{\partial \hat{x}}\right|_{\hat{x}=\hat{x}_{k-1}^{+}} \\
H_{k} & =\left.\frac{\partial h\left(\hat{x}, u_{k-1}\right)}{\partial \hat{x}}\right|_{\hat{x}=\hat{x}_{k}^{-}}
\end{aligned}
$$

where $K_{k}$ is usually denoted as the Kalman gain. The following initializing conditions are applied to the state estimates and to the error covariance:

$$
\begin{aligned}
\hat{x}_{0}^{+} & =E\left[x_{0}\right] \\
P_{0}^{+} & =E\left[\left(x_{0}-\hat{x}_{0}^{+}\right)\left(x_{0}-\hat{x}_{0}^{+}\right)^{T}\right]
\end{aligned}
$$

where $E[\cdot]$ is the expected value.

\section{SMO Design}

The design of an SMO for the system (9)-(10) has been carried out according to the methodology reported in [33].

Considering the output vector components, it can be easily verified that the relative degree $r$ of the outputs are:

$$
r_{y}=2, \quad r_{P_{L}}=1, \quad r_{v_{e}}=2
$$


It can be also verified that the sum of the output relative degrees is 5 , i.e. the dimension of whole state space, meaning that, given the output vector $z(t)$, there are no hidden dynamics in the system. Moreover, the conditions for the design of the SMO are also satisfied. Therefore the SMO is obtained by combining an optimal linear state observer and a SMO

$$
\dot{\hat{x}}=f(\hat{x})+g(\hat{x}) u+L(h(\hat{x})-z)+\Gamma e
$$

where $\Gamma=\operatorname{diag}\left[\begin{array}{lll}\eta_{1} & \cdots & \eta_{5}\end{array}\right]$ is the diagonal matrix of positive SMO gains and

$$
e=\left[\begin{array}{c}
e_{1} \\
e_{2} \\
e_{3} \\
e_{4} \\
e_{5}
\end{array}\right]=\operatorname{sign}\left[\begin{array}{c}
y-\hat{x}_{1} \\
\eta_{1} e_{1} \\
P_{L}-\hat{x}_{3} \\
v_{e}-\hat{x}_{4} \\
\eta_{4} e_{4}
\end{array}\right]
$$

is the vector of SMO nonlinear error functions.

The linear observer gain, $L$, has been designed considering the linear part of the system (8) only and computing the solution of the Continuous-time Algebraic Riccati Equation (CARE) with identity weight matrix. Therefore, the equations of the considered linear system are

$$
\begin{aligned}
\dot{x} & =A_{o} x+B_{o} u \\
z & =C_{o} x
\end{aligned}
$$

where

$$
A_{o}=\left[\begin{array}{ccccc}
0 & 1 & 0 & 0 & 0 \\
0 & -\frac{b}{m} & \frac{A_{p}}{m} & 0 & 0 \\
0 & -\frac{2 \beta A_{p}}{V_{0}} & 0 & 0 & 0 \\
0 & 0 & 0 & 0 & 1 \\
0 & 0 & 0 & -\omega_{n v}^{2} & -2 \epsilon_{v} \omega_{n v}
\end{array}\right], \quad B_{o}=g(x), \quad C_{o}=\left[\begin{array}{ccccc}
1 & 0 & 0 & 0 & 0 \\
0 & 0 & 1 & 0 & 0 \\
0 & 0 & 0 & 1 & 0
\end{array}\right]
$$

Assuming that the state estimation error goes to zero, the estimation of the disturbance acting on the system can be expressed as

$$
\hat{\varphi}(x, t)=\dot{\hat{x}}-f(\hat{x})-g(\hat{x}) u-L(h(\hat{x})-z)-\Gamma e
$$

\section{HSMO Design}

The design of the HSMO for the system (8) exploits the approach proposed in [26]. Therefore, this HSMO also presents the capability of unknown disturbance estimation. Given the properties about the output relative degrees already discussed in the design of the SMO carried out in Sec. 4, it follows that the HSMO is able to provide, in finite-time, an estimation for both the state and the external disturbances, i.e.

$$
\begin{aligned}
& \lim _{t \rightarrow \infty}\|\hat{x}(t)-x(t)\|=0 \\
& \lim _{t \rightarrow \infty}\|\hat{\varphi}(t)-\varphi(t)\|=0
\end{aligned}
$$

Since no hidden dynamics is present in the system, as described in Sec. 4, the system (8) can be rewritten in Brunowsky canonical form as:

$$
\dot{\xi}=\Lambda \xi+\Phi(\xi)+\varphi(\xi)+g(\xi) u
$$

where $\xi(t)=\hat{x}(t)$ is the state estimation and

$$
\Lambda=\left[\begin{array}{lllll}
0 & 1 & 0 & 0 & 0 \\
0 & 0 & 0 & 0 & 0 \\
0 & 0 & 0 & 0 & 0 \\
0 & 0 & 0 & 0 & 1 \\
0 & 0 & 0 & 0 & 0
\end{array}\right], \quad \Phi(\xi)=\left[\begin{array}{c}
0 \\
-\frac{b}{m} \dot{\hat{y}}+\frac{A_{p} \hat{P}_{L}}{m} \\
-\frac{2 \beta A_{P}}{V_{0}} \dot{\hat{y}}+\frac{2 \beta k_{q} \hat{v}_{e} \sqrt{P_{s}-\left|\hat{P}_{L}\right|}}{V_{0}} \\
-\omega_{n v}^{2} \hat{v}_{e}-2 \epsilon_{v} \omega_{n v} \dot{\hat{v}}_{e}
\end{array}\right], \quad \varphi(x(t))=\left.\varphi(x(t), t)\right|_{\eta(t)=[0,0,0]^{T}}
$$




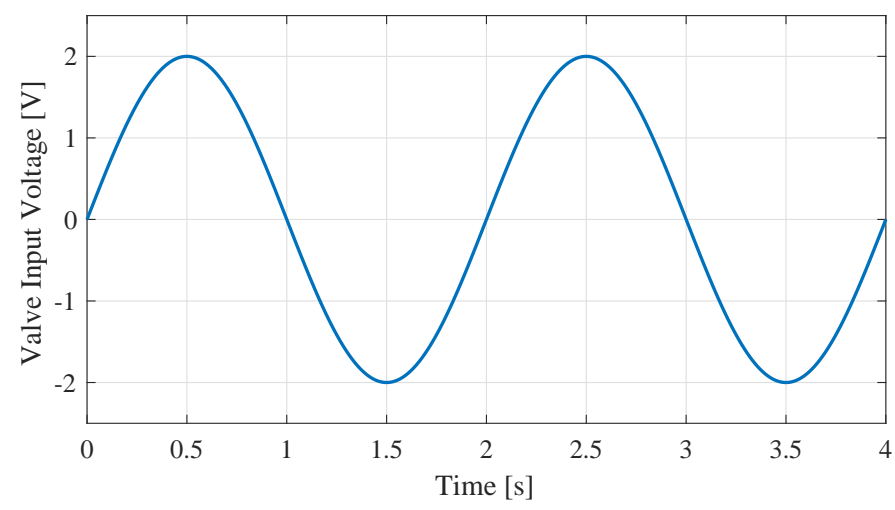

Figure 2: Valve input voltage adopted for all the tests.

The derivative of the observer state $\xi_{j}^{\{i\}}(t), \forall i \in[1,2,3], \forall j=0, \ldots, r_{i}-1$, being $r_{i}$ the relative degree of the $i$-th output, can be estimated in finite-time from the measured outputs $z_{i}=h_{i}(x)$ by means of a high-order sliding-mode differentiator [34]:

$$
\begin{aligned}
j & \in \\
v_{-1}^{\{i\}} & =z_{i} \\
& \cdots \\
\dot{\sigma}_{j}^{\{i\}} & =v_{j}^{\{i\}} \\
e_{j}^{\{i\}} & =\sigma_{j}^{\{i\}}-v_{j-1}^{\{i\}} \\
v_{j}^{\{i\}} & =-\nu_{j}^{\{i\}} \delta^{\{i\} 1 /\left(r_{i}-j+1\right)} \\
& \\
& \left.\cdots e_{j}^{\{i\}}\right|^{\left(r_{i}-j\right) /\left(r_{i}-j+1\right)} \operatorname{sign}\left(e_{j}^{\{i\}}\right)+\sigma_{j+1}^{\{i\}} \\
\dot{\sigma}_{r_{i}}^{\{i\}} & =-\nu_{r_{i}}^{\{i\}} \delta^{\{i\}} \operatorname{sign}\left(e_{r_{i}}^{\{i\}}\right)
\end{aligned}
$$

where, according to [34],

$$
\nu_{j}^{\{1,3\}}=[3,1.5,1.1], \quad \nu_{j}^{\{2\}}=[1.5,1.1]
$$

In eqs. (35), the coefficients $\delta^{\{i\}}$ are scaling factors that can be use to adjust the convergence rate of the observer [28]. It follows that

$$
\xi_{j+1}^{\{i\}}=\sigma_{j}^{\{i\}}, j \in 0, \ldots, r_{i}-1, \quad \dot{\xi}_{r_{i}}^{\{i\}}=\sigma_{r_{i}}^{\{i\}}
$$

Since finite-time exact estimates of $\xi$ are available via the higher-order sliding-mode differentiator (35), an asymptotic estimation of the disturbance input vector can be provided by means of

$$
\hat{\varphi}(\xi, t)=\dot{\xi}-\Lambda \xi-\Phi(\xi)-g(\xi) u
$$

\section{Simulations}

In order to evaluate the benefits of the proposed estimator, simulations have been carried out on the mathematical model of the hydraulic actuator (8). The model parameters have been determined by means of an identification procedure [31], and are reported in Tab. 1.

It is worth noting that, during the simulations, an asymmetric dead-zone in the valve has been considered in the simulation model $\left(v_{e p}=0.43 \mathrm{~V} ; v_{e n}=0.21 \mathrm{~V}\right)$, and that different valve gains have been assumed in the positive and negative working regions. Also a small valve leakage around the rest position has been considered in the valve simulation model, while an ideal valve with no dead-zone has been assumed in the observer design. Another important consideration related to the experimental implementation of the system is that no information is available from the valve manufacturer about the relation between the valve output position signal and the effective valve spool 
Table 1: Nominal parameters of the hydraulic actuator.

\begin{tabular}{|l|c|c|c|}
\hline Description & Symbol & Unit & Value \\
\hline Piston mass & $m$ & $\mathrm{~kg}$ & 440 \\
\hline Piston viscous friction coeff. & $b$ & $\mathrm{~N} \mathrm{~s} \mathrm{~m}^{-1}$ & 23555 \\
\hline Piston static friction force & $F_{c 0}$ & $\mathrm{~N}$ & 950 \\
\hline Piston Coulomb friction force & $F_{c}$ & $\mathrm{~N}$ & 950 \\
\hline Guide static friction coeff. & $\mu_{0}$ & & 0.01 \\
\hline Guide Coulomb friction coeff. & $\mu$ & & 0.01 \\
\hline Piston area & $A_{p}$ & $\mathrm{~m}^{2}$ & 0.01 \\
\hline Centered camera volume & $V_{0}$ & $\mathrm{~m}^{3}$ & 0.004 \\
\hline Bulk modulus & $\beta$ & $\mathrm{Pa}^{3}$ & $1 \mathrm{e} 9$ \\
\hline Nominal valve gain & $k_{q}$ & $\mathrm{~m}^{3} /\left(\mathrm{s} \mathrm{V} \mathrm{Pa}^{\frac{1}{2}}\right)$ & $6 \mathrm{e}-7$ \\
\hline dead-zone center gain & $k_{q 0}$ & $\mathrm{~m}^{3} /\left(\mathrm{s} \mathrm{V} \mathrm{Pa}^{\frac{1}{2}}\right)$ & $1 \mathrm{e}-12$ \\
\hline dead-zone positive gain & $k_{q p}$ & $\mathrm{~m}^{3} /\left(\mathrm{s} \mathrm{V} \mathrm{Pa}^{\frac{1}{2}}\right)$ & $6.15 \mathrm{e}-7$ \\
\hline dead-zone negative gain & $k_{q n}$ & $\mathrm{~m}^{3} /\left(\mathrm{s} \mathrm{V} \mathrm{Pa}^{\frac{1}{2}}\right)$ & $5.86 \mathrm{e}-7$ \\
\hline Spool position bias & $v_{e 0}$ & $\mathrm{~V}$ & 0.01 \\
\hline Input gain & $k_{e}$ & & 0.49 \\
\hline Valve natural frequency & $\omega_{n v}$ & $\mathrm{~s}^{-1}$ & 152 \\
\hline Valve damping coeff. & $\epsilon_{v}$ & & 0.92 \\
\hline Supply pressure & $P_{s}$ & $\mathrm{~Pa}_{e p}$ & $6 \mathrm{e} 6$ \\
\hline Pos. valve dead-zone voltage & $v_{e p}$ & 0.43 \\
\hline Neg. valve dead-zone voltage & $v_{e n}$ & $\mathrm{~V}$ & 0.21 \\
\hline
\end{tabular}

position. Therefore, the valve spool position and velocity will be expressed in Volts to reflect the output position signal provided by the valve itself.

Simulations have been performed in two different conditions, assuming perfect knowledge of the measured outputs during the first test and considering the presence of measurement noise in the second. The performance of the SMO and the HSMO will be evaluated through a comparison with the EKF estimator and the simulated state.

The simulated results have been obtained by means of open loop tests employing a sine wave as input, characterized by a frequency low enough to better appreciate the effects of the dead-zone. For all the tests reported in this paper we assumed $u(t)=2 \sin (\pi t)$, as seen in Fig. 2. The parameters adopted for the observers are the same of the hydraulic actuator model reported in Tab. 1, but the piston friction, the valve dead-zone and the valve spool bias have been neglected in the observer design. Therefore, these quantities, i.e. the piston friction, the valve dead-zone and the valve spool bias, are considered as unknown disturbance inputs to be reconstructed by the observers.

\subsection{Simulation with ideal measurements}

The first simulation has been carried out by assuming perfect knowledge of the outputs (i.e. no measurement noise) to evaluate the capabilities of the three observers, the EKF, the SMO and the HSMO, to correctly estimate the system state as well as the disturbances, i.e. the friction acting on the hydraulic cylinder, the valve dead-zone and its bias.

Even if no noise is assumed to act on the system during this test, the EKF has been implemented assuming the process noise covariance matrix $L_{k}$ and the output noise covariance matrix $M_{k}$ equal to the identity matrix of proper dimensions. The EKF has been selected since it represents a well-known reference in the field of state estimation. Moreover, the EKF parameters are the same adopted in [17] to allow a direct comparison between the results and then to validate the proposed approach.

The SMO gains have been selected according to the design procedure reported in [27] and [33] and trying to achieve a good trade-off between observer estimation accuracy and limited estimation chattering due to the switching nature of the observer. The gains adopted during the simulations are reported in Tab. 2. On the other hand, the scaling factors of the HSMO are reported in Tab. 3 and they have been designed as a suitable trade-off between estimation error convergence speed and sensitivity to measurement noise. Both for the SMO and the HSMO, several parameters sets have been tested in simulation to find the aforementioned observer performance trade-off, but for the sake of brevity the response provided by the selected parameter set are reported only for the two observers. It is worth 


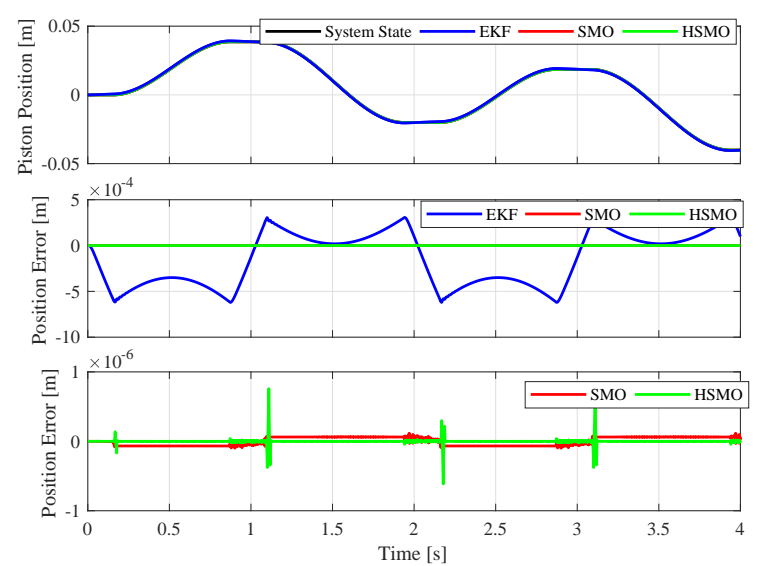

(a) Piston Position. Top plot: simulated and estimated piston position. Middle plot: comparison between the estimation error of the three observers. Bottom plot: comparison between the estimation error of the the SMO and the HSMO.

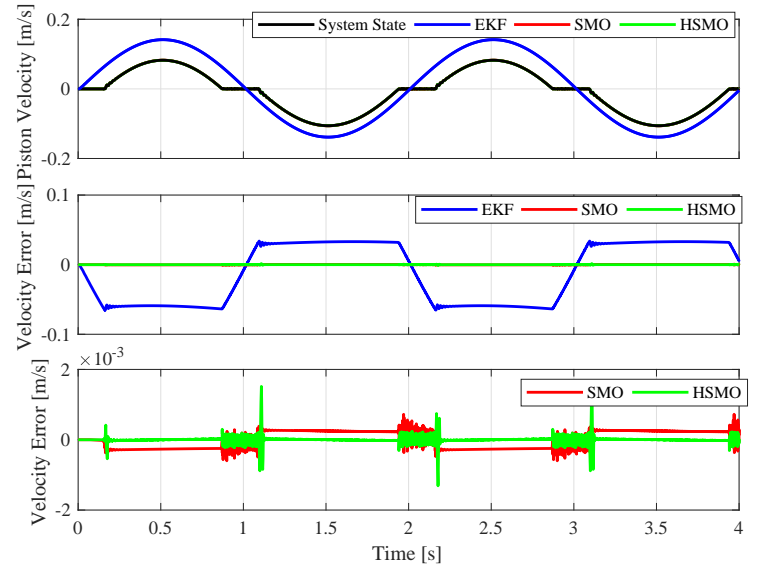

(b) Piston Velocity. Top plot: simulated and estimated piston velocity. Middle plot: comparison between the estimation error of the three observers. Bottom plot: comparison between the estimation error of the SMO and the HSMO.
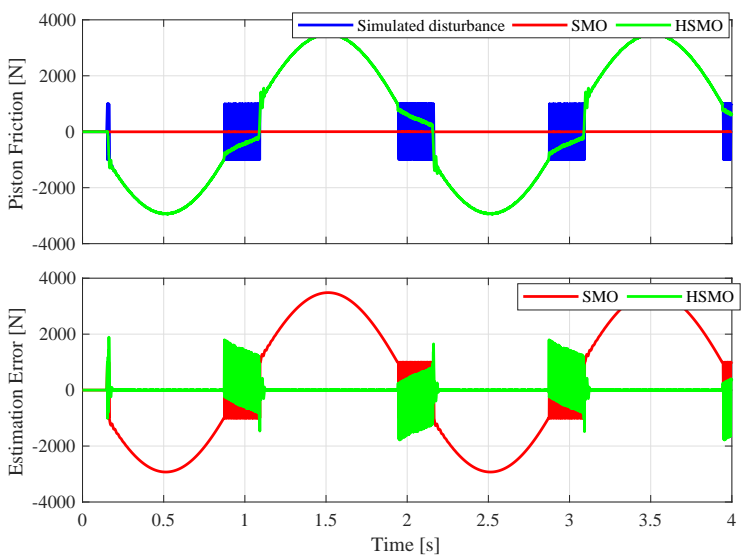

(c) Piston Friction. Top plot: friction acting on the piston during the simulation and the one estimated by observers. Bottom plot: comparison between the estimation error of the the SMO and the HSMO.

Figure 3: Piston position, velocity and friction during simulation (black), data estimated by the EKF (blue), by the SMO (red) and by the HSMO (green).

noting that this is also one of the main purpose of simulations, i.e. to ease the test of different parameter sets and find a suitable compromise between conflicting design specifications limiting time consuming experimental tests.

The simulation results in case of no noise in the measurements are shown in Fig. 3, 4 and 5. In particular, Fig. 3 reports the piston position, velocity and friction. More in detail, Fig. 3(a) shows the simulated and estimated piston position by the EKF, the SMO and the HSMO together with the comparison between the estimation errors, to highlight the very large error of the EKF with respect to the SMO and the HSMO. The bottom plot reports the comparison between the estimation error of the the SMO and the HSMO only, to highlight the better performance of the HSMO with respect to the SMO.

Figure 3(b) reports the simulated and estimated piston velocity by the three observers, as well as the comparison between the estimation errors to highlight the very large error of the EKF with respect to the SMO and the HSMO. In the bottom plot, the comparison between the estimation error of the SMO and the HSMO are reported to highlight the better performance of the HSMO with respect to the SMO.

Table 2: Gains of the SMO.

\begin{tabular}{|c|c|c|c|c|}
\hline$\eta_{1}$ & $\eta_{2}$ & $\eta_{3}$ & $\eta_{4}$ & $\eta_{5}$ \\
\hline 20 & 45.5 & 60000 & 0.2 & 2 \\
\hline
\end{tabular}




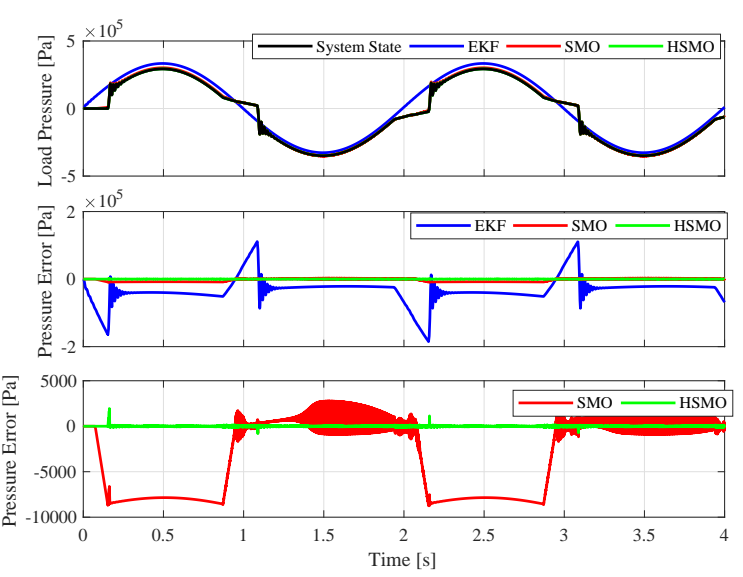

(a) Pressure inside the piston chamber. Top plot: simulated and estimated piston pressure. Middle plot: comparison between the estimation error of the three observers. Bottom plot: comparison between the estimation error of the SMO and the HSMO only.

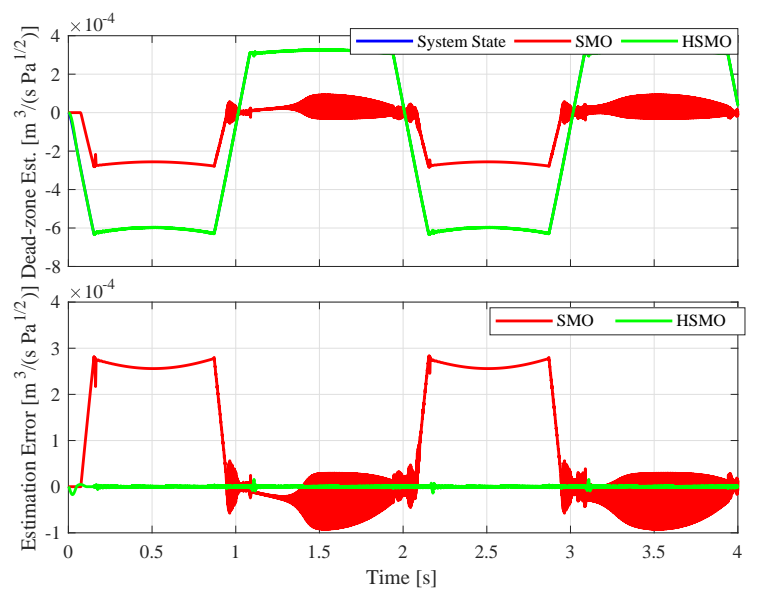

(b) Valve dead-zone. Top plot: valve dead-zone during the simulation and the one estimated by the SMO and the HSMO. Bottom plot: comparison between the estimation error of the SMO and the HSMO.

Figure 4: Pressure inside the piston chamber and valve dead-zone during simulation (black) and estimated by the EKF (blue), by the SMO (red) and by the HSMO (green).

Finally, Fig. 3(c) shows the friction acting on the piston during the simulation and the one estimated by the SMO and the HSMO (the EKF does not provide any disturbance estimation). In particular, Fig. 3(c) reports the friction acting on the piston during the simulation and the one estimated by the SMO and the HSMO (note that the friction estimated by the SMO is almost zero). The comparison between the estimation error of the the SMO and the HSMO is reported in the bottom plot to highlight the fact that the SMO does not provide a reliable estimation of the piston friction.

The reason why the SMO shows a poor friction estimation can be ascribed to the fact that, differently from the HSMO that is based on the concept of high-order differentiator [34], the SMO state estimation dynamics cannot guarantee a proper relation between the different components of the state vector. This in turn results into poor and strongly state dependent disturbance estimation performance.

The pressure in the piston chamber and the effect of the valve dead-zone are reported in Fig. 4. In particular, Fig. 4(a) reports the simulated and estimated piston pressure and the comparison between the estimation error of the EKF, the SMO and the HSMO, to highlight the very large error of the EKF with respect to the SMO and the HSMO. In the bottom plot, the comparison between the estimation error of the the SMO and the HSMO only is reported, to highlight the better performance of the HSMO with respect to the SMO.

For what related to the valve dead-zone, Fig. 4(b) reports the valve dead-zone during the simulation and the one estimated by the SMO and the HSMO in the top plot, while in the bottom plot, the comparison between the estimation error of the SMO and the HSMO, to highlight the fact that the SMO does not provide a reliable estimation of the piston friction, while the HSMO estimation error is close to zero.

The simulation data related to the valve are reported in Fig. 5. In particular, Fig. 5(a) shows the spool position and its estimation together with the comparison between the estimation errors of the EKF, the SMO and the HSMO, to highlight the very large error of the EKF with respect to the SMO and the HSMO.

Figure 5(b) reports the spool velocity and the one estimated by the EKF, the SMO and the HSMO in the top plot, while in the bottom plot, the comparison between the estimation error of the EKF, the SMO and the HSMO, in this case the three observer performance are comparable.

Finally, Fig. 5(c) shows the valve input bias during the simulation and the one estimated by the SMO and the HSMO (the EKF does not provide any disturbance estimation). In particular, Fig. 3(c) reports the input bias of the valve during the simulation and the one estimated by the SMO and the HSMO together with the comparison between their estimation errors, to highlight the fact that the SMO does not provide a reliable estimation of the

Table 3: Scaling coefficients of the HSMO.

\begin{tabular}{|c|c|c|}
\hline$\delta^{\{1\}}$ & $\delta^{\{1\}}$ & $\delta^{\{1\}}$ \\
\hline 220 & 60000 & 1000 \\
\hline
\end{tabular}



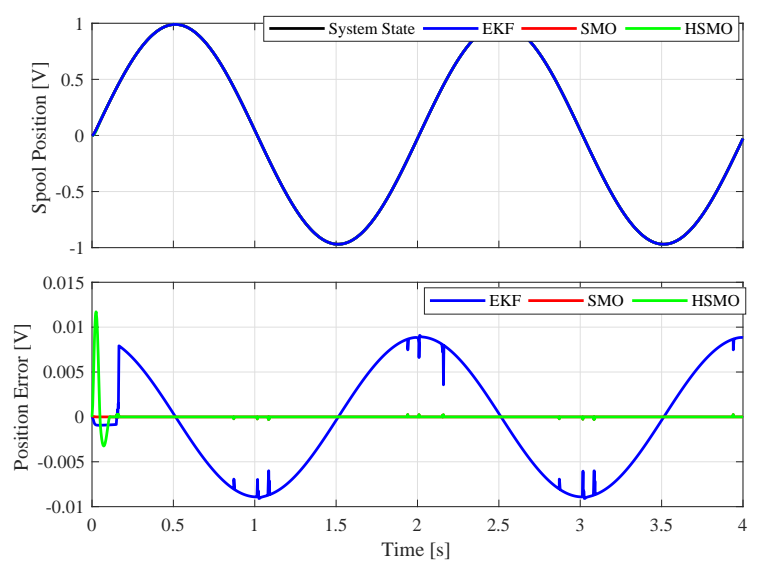

(a) Spool position. Top plot: spool position and its estimation by the three observers. Bottom plot: comparison between the estimation error of the three observers.
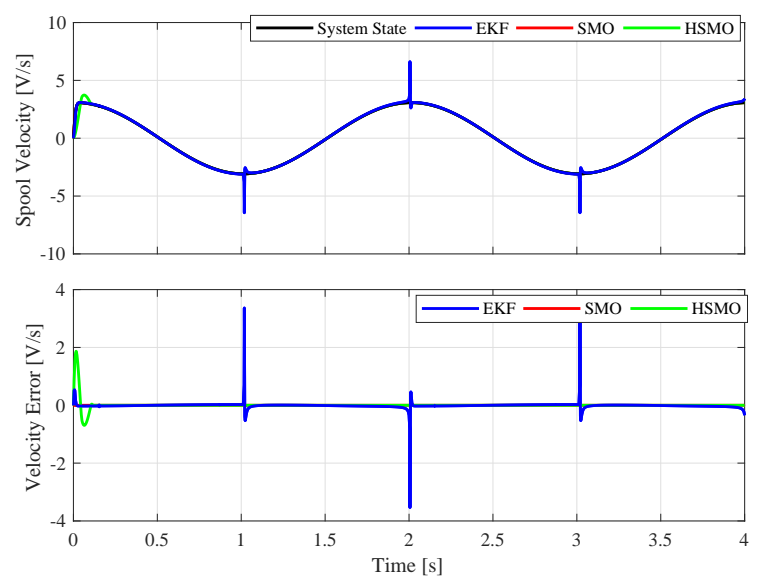

(b) Spool velocity. Top plot: spool velocity simulation and estimation. Bottom plot: comparison between the estimation error of the three observers.
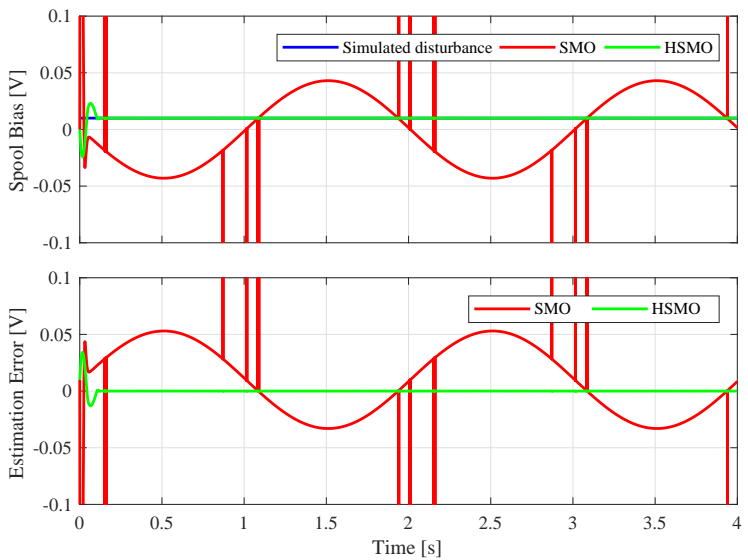

(c) Valve input bias. Top plot: valve input bias during the simulation and the one estimated by the SMO and the HSMO. Bottom plot: comparison between the estimation error of the SMO and the HSMO.

Figure 5: Valve position, velocity and input bias during simulation (black) and estimated by the EKF (blue), by the SMO (red) and by the HSMO (green).

valve input bias.

To summarize, the performance of the designed observers in terms RMS value of the state and disturbance estimation errors are reported in Tab. 4 and 5 respectively. These data confirm that the HSMO performs better than the other observers in almost all the cases.

\subsection{Simulation with noise in the measurements}

The second simulation has been carried out to evaluate the capabilities of the three observers in case the output measurements are affected by noise. The parameters of the noise acting on each output are reported in Tab. 6 .

As in the previous case, the EKF, the SMO and the HSMO estimate the system state as well as the disturbances, i.e. the friction acting on the hydraulic cylinder, the valve dead-zone and its bias.

Table 4: RMS value of the state estimation errors.

\begin{tabular}{|l|c|c|c|c|c|}
\hline & $y$ & $\dot{y}$ & $P_{L}$ & $v_{e}$ & $\dot{v}_{e}$ \\
\hline EKF & $1.6503 \mathrm{e}-6$ & $2.2145 \mathrm{e}-4$ & 278.22 & $2.9039 \mathrm{e}-5$ & $4.0586 \mathrm{e}-4$ \\
\hline SMO & $2.8377 \mathrm{e}-10$ & $1.1495 \mathrm{e}-6$ & 25.950 & $1.5413 \mathrm{e}-7$ & $2.4494 \mathrm{e}-6$ \\
\hline HSMO & $2.1673 \mathrm{e}-10$ & $5.4116 \mathrm{e}-7$ & 0.6121 & $4.3315 \mathrm{e}-6$ & $7.1518 \mathrm{e}-4$ \\
\hline
\end{tabular}




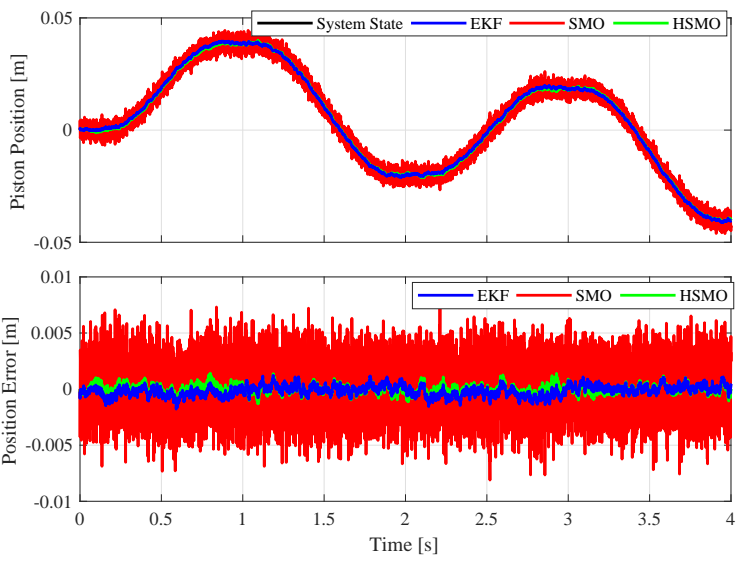

(a) Piston Position. Top plot: simulated and estimated piston position. Bottom plot: comparison between the estimation errors.
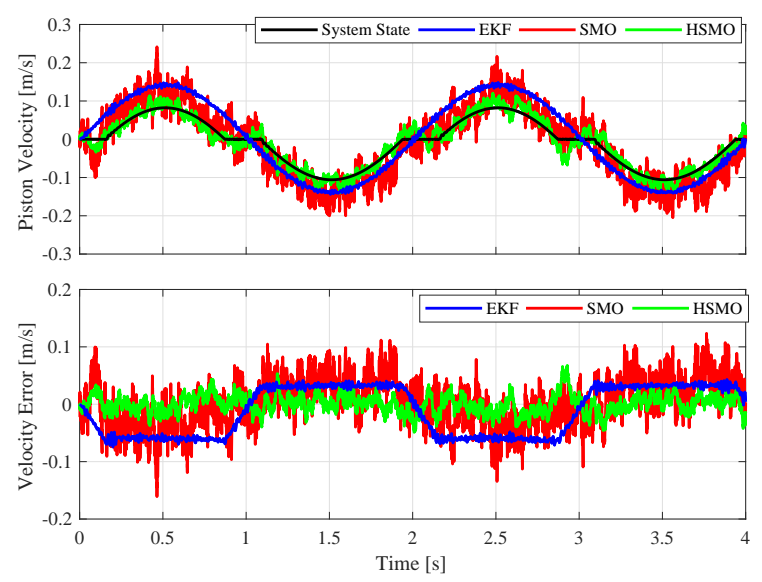

(b) Piston Velocity. Top plot: simulated and estimated piston velocity. Bottom plot: comparison between the estimation errors.

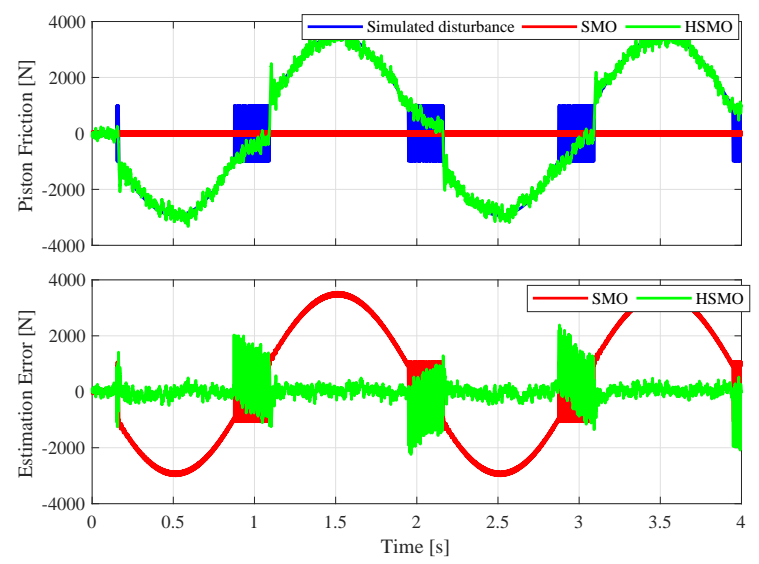

(c) Piston Friction. Top plot: simulated and estimated friction acting on the piston. Bottom plot: comparison between the estimation errors.

Figure 6: Piston position, velocity and friction during simulation in case of noise in the measurements (black), data estimated by the EKF (blue), by the SMO (red) and by the HSMO (green).

Also in this case, the EKF has been implemented assuming the process noise covariance matrix $L_{k}$ and the output noise covariance matrix $M_{k}$ equal to the identity matrix on opportune dimension. The SMO gains and HSMO scaling factors are the same adopted during the previous simulation tests and they are reported in Tab. 2 and 3 respectively.

The results of this simulation are shown in Fig. 6,7 and 8. In particular,Fig. 6 reports the piston position, velocity and friction. More in detail, Fig. 6(a) shows the simulated piston position and the position estimated by the EKF, the SMO and the HSMO, together with the comparison between the estimation errors of the three observers. In this case, it is possible to see from the plot of the piston position estimation error reported in the bottom plot of Fig. 6(a) that the EKF and the HSMO perform similarly, while the SMO is much more sensitive to the measurement noise.

Figure 6(b) reports the simulated piston velocity and the three observer estimations, as well as the comparison between their estimation errors, to highlight the very large error of the EKF with respect to the SMO and the HSMO. From the piston velocity estimation error reported in the bottom plot of Fig. 6(b) it can be seen that the EKF is less sensitive to the noise, but in general the HSMO provides a better estimation of the state.

Table 5: RMS value of the disturbance estimation errors.

\begin{tabular}{|l|c|c|c|}
\hline & Piston Friction & Load Pressure & Valve Bias \\
\hline SMO & 11.067 & $8.4590 \mathrm{e}-7$ & 0.0016 \\
\hline HSMO & 1.6026 & $9.5660 \mathrm{e}-9$ & $1.3839 \mathrm{e}-5$ \\
\hline
\end{tabular}



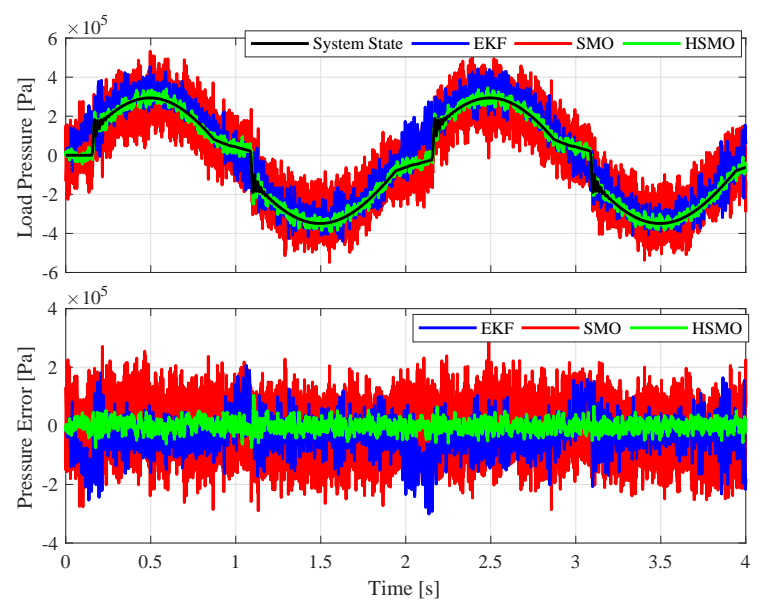

(a) Pressure inside the piston chamber. Top plot: simulated and estimated piston pressure. Bottom plot: comparison between the estimation errors.

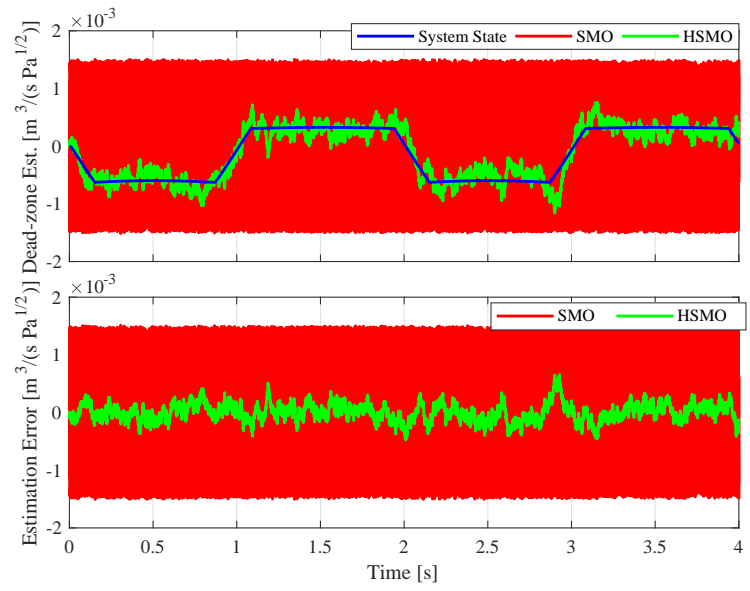

(b) Valve dead-zone. Top plot: valve dead-zone during the simulation and the one estimated by the SMO and the HSMO. Bottom plot: comparison between the estimation error of the SMO and the HSMO.

Figure 7: Pressure inside the piston chamber and valve dead-zone during simulation in case of noise in the measurements (black) and estimated by the EKF (blue), by the SMO (red) and by the HSMO (green).

Finally, Fig. 6(c) shows the friction acting on the piston during the simulation and the one estimated by the SMO and the HSMO (the EKF does not provide any disturbance estimation). In the top plot, the friction acting on the piston during the simulation and the one estimated by the SMO and the HSMO is reported (note that the friction estimated by the SMO is almost zero). The bottom plot shows the comparison between the estimation error of the the SMO and the HSMO, to highlight the fact that the SMO does not provide a reliable estimation of the piston friction while, despite the noise, the HSMO is still able to provide a reliable disturbance estimation.

The pressure in the piston chamber and the effect of the valve dead-zone are reported in Fig. 7. Figure 7(a) reports, in the top plot, the simulated piston pressure and the one estimated by the EKF, the SMO and the HSMO, while, in the bottom plot, the comparison between the estimation errors highlights the very large error of the EKF with respect to the SMO and the HSMO.

It is possible to see from the plot of the chamber pressure estimation error reported in the bottom plot of Fig. 7(a) that the HSMO performs better than the other observers, and the particular that the SMO is much more sensitive to the measurement noise with respect to the others. For what related to the valve dead-zone, Fig. 7(b) reports the valve dead-zone during the simulation and the one estimated by the SMO and the HSMO in the top plot, while, in the bottom plot, the comparison between the estimation errors of the two observers is shown. Also in this case, the SMO is not able to provide a reliable disturbance estimation due also to the presence of the measurement noise.

Figure 8 reports the simulation of the valve. In particular, in Fig. 8(a) the valve position and the position estimated by the EKF, the SMO and the HSMO are shown together with the comparison between the estimation errors of the three observers.

From the bottom plot, it possible to see that in this case the EKF provides a more noisy state estimation, while the SMO provides even better estimation than the HSMO. Similar results can be seen in Fig. 8(b) about the spool velocity, in the top plot, the spool velocity and the one estimated by the EKF, the SMO and the HSMO are reported, while the bottom plot reports the comparison between their estimation errors. As for the valve position, also in this case it possible to see that the SMO provides even better than the HSMO, while the EKF provides a noisy state estimation.

Finally, Fig. 8(c) shows, in the top plot, the valve input bias during the simulation and the one estimated by the SMO and the HSMO (the EKF does not provide any disturbance estimation), while, in the bottom plot, the comparison between the estimation error of the SMO and the HSMO is reported. Also in this case, the HSMO shows

Table 6: Parameters of the noise for each output measurement.

\begin{tabular}{|l|c|c|c|}
\hline Output & $y$ & $P_{L}$ & $v_{e}$ \\
\hline Mean Value & 0 & 0 & 0 \\
\hline Covariance & $5 \mathrm{e}-3^{2}$ & $1 \mathrm{e} 5^{2}$ & $1 \mathrm{e}-1^{2}$ \\
\hline
\end{tabular}




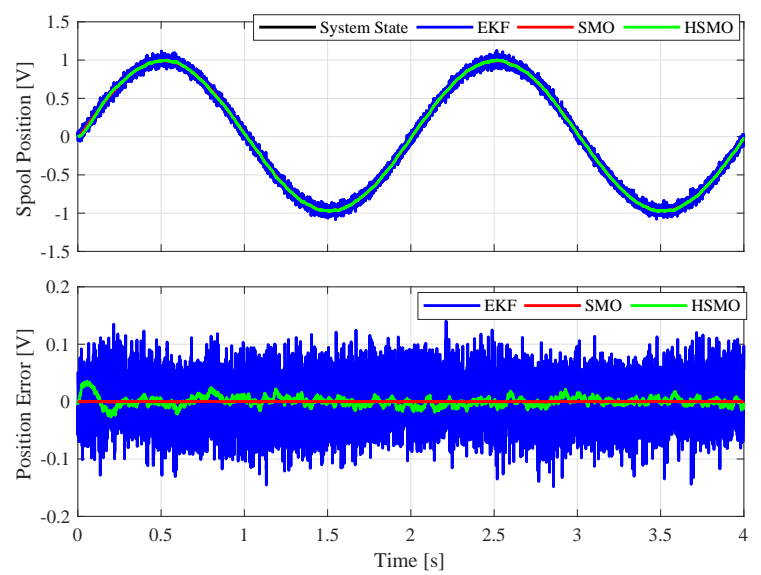

(a) Spool position. Top plot: simulated valve position and position estimation. Bottom plot: comparison between the estimation errors of the three observers.
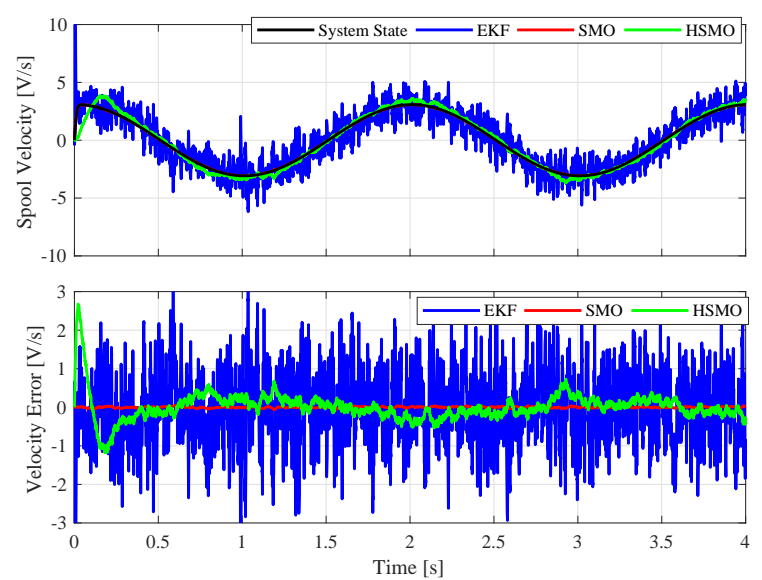

(b) Spool velocity. Top plot: simulated and estimated spool velocity. Bottom plot: comparison between the estimation errors.

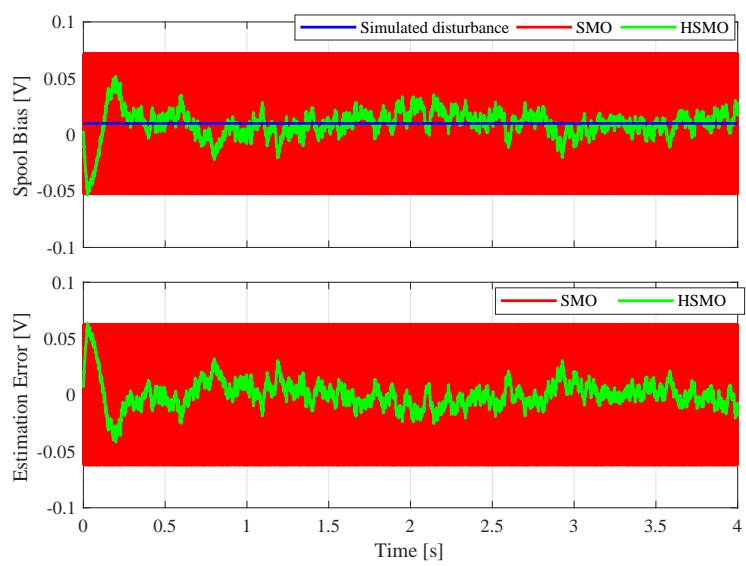

(c) Valve input bias. Top plot: simulated and estimated input bias of the valve. Bottom plot: comparison between the estimation error of the SMO and the HSMO.

Figure 8: Valve position, velocity and input bias during simulation in case of noise in the measurements (black) and estimated by the EKF (blue), by the SMO (red) and by the HSMO (green).

the advantage of providing a reliable disturbance estimation even in presence of noise.

The performance of the designed observers in terms RMS value of the state and disturbance estimation errors in case of noise in the outputs are reported in Tab. 7 and 8 respectively. These data confirm that the HSMO performs better than the other observers in almost all the cases.

\section{Experimental Results}

Experimental tests have been performed on the hydraulic system test-bed to verify the effectiveness of the proposed approach for the observation of the system state. For the ease of comparison, the experiment reported in this paper replicates the same conditions evaluated during the simulations. A longer time interval will be shown in the experimental data plots to show in a better way the effectiveness of the method in real conditions.

Table 7: RMS value of the state estimation errors in case of noise in the output measurements.

\begin{tabular}{|l|c|c|c|c|c|}
\hline & $y$ & $\dot{y}$ & $P_{L}$ & $v_{e}$ & $\dot{v}_{e}$ \\
\hline EKF & $2.5083 \mathrm{e}-6$ & $2.2293 \mathrm{e}-4$ & 387.18 & $1.7428 \mathrm{e}-4$ & 0.0052 \\
\hline SMO & $9.5067 \mathrm{e}-6$ & $1.9305 \mathrm{e}-4$ & 356.11 & $1.1722 \mathrm{e}-6$ & $8.1682 \mathrm{e}-5$ \\
\hline HSMO & $1.9627 \mathrm{e}-6$ & $7.6326 \mathrm{e}-5$ & 90.984 & $4.0858 \mathrm{e}-5$ & 0.0020 \\
\hline
\end{tabular}




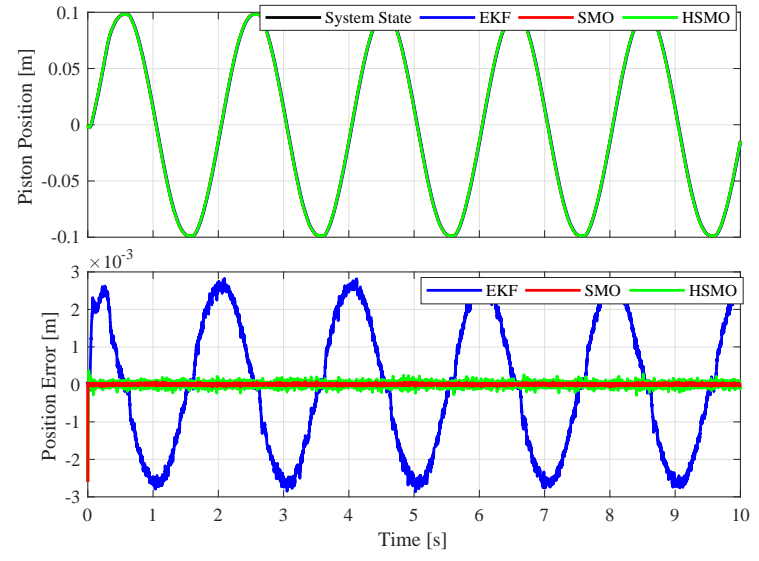

(a) Piston Position. Top plot: the piston position and its estimation by the three observers.Bottom plot: comparison between the estimation errors.
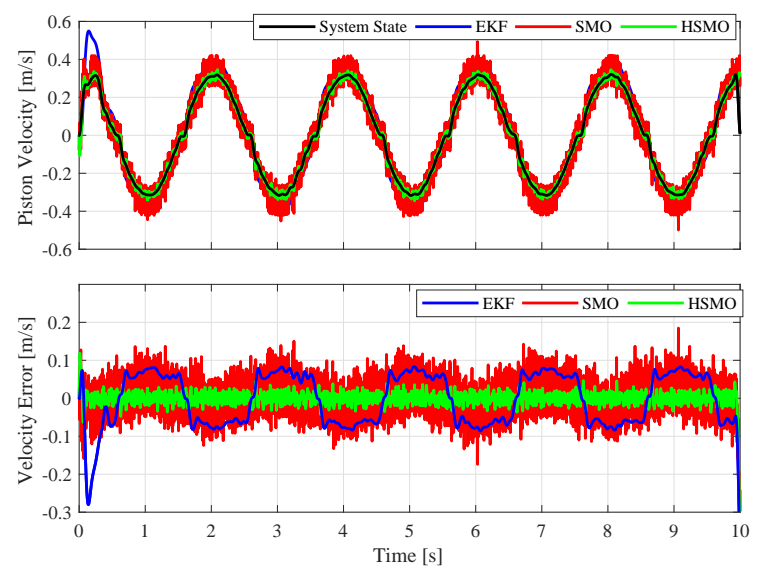

(b) Piston Velocity. Top plot: piston velocity and estimation by the three observers. Bottom plot: comparison between the estimation errors.

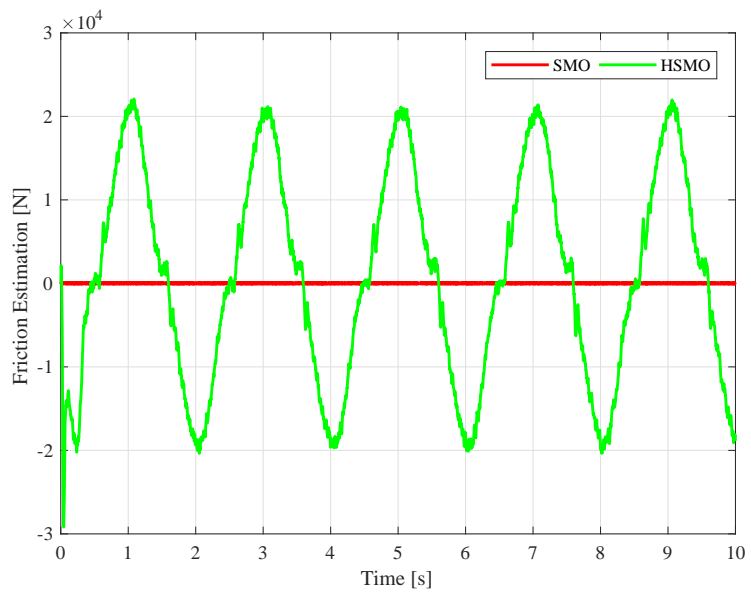

(c) Friction acting on the piston estimated by the SMO and the HSMO.

Figure 9: Piston position, velocity and friction during the experiment (black), data estimated by the EKF (blue), by the SMO (red) and by the HSMO (green).

As for the previous simulation tests, both the EKF, the SMO and the HSMO have been evaluated and compared to show the benefits of the different observers. In particular, the evaluated observers are the same used during the simulation, therefore they are designed on the base of the nominal system parameters reported in Tab. 1. The SMO gains and HSMO scaling factors are the same adopted during the previous simulation tests and they are reported in Tab. 2 and 3 respectively.

The experimental setup is equipped with sensors that provide the same output information assumed during the simulations, i.e. the piston position, pressure and spool position. As mentioned in case of the simulation tests, no information is available from the valve manufacturer about the relation between the valve output position signal and the effective valve spool position. Therefore, the valve spool position and velocity will be expressed in volts to reflect the output position signal provided by the valve itself. For the comparison with the piston and spool velocities estimated by the observers, these data have been reconstructed from the respective position information through digital filtering. During the experiments, no data can be obtained about the disturbance affecting the system, therefore any consideration about their quality can be performed in relation to simulation results only.

Table 8: RMS value of the disturbance estimation errors in case of noise in the output measurements.

\begin{tabular}{|l|c|c|c|}
\hline & Piston Friction & Load Pressure & Valve Bias \\
\hline SMO & 11.082 & $5.6295 \mathrm{e}-6$ & 0.0152 \\
\hline HSMO & 1.8828 & $7.4070 \mathrm{e}-7$ & $6.2572 \mathrm{e}-5$ \\
\hline
\end{tabular}



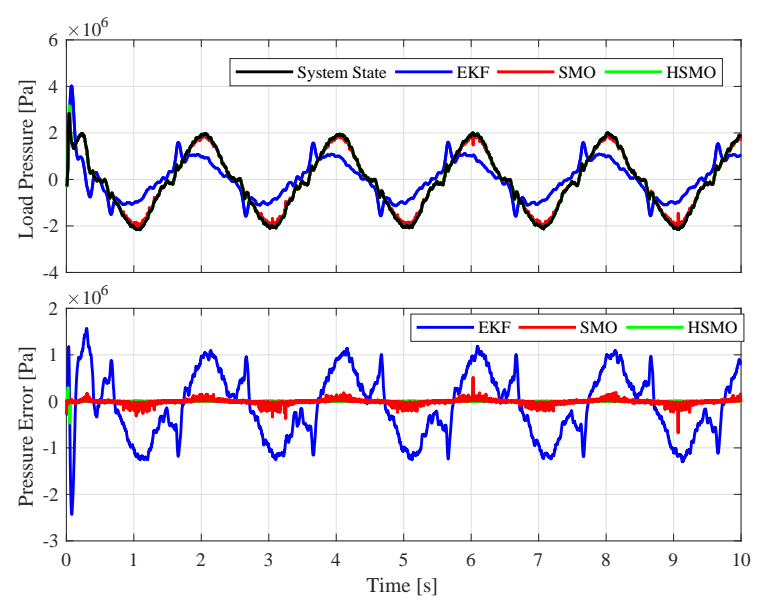

(a) Pressure inside the piston chamber. Top plot: piston pressure and estimation by the EKF, the SMO and the HSMO. Bottom plot: comparison between estimation errors.

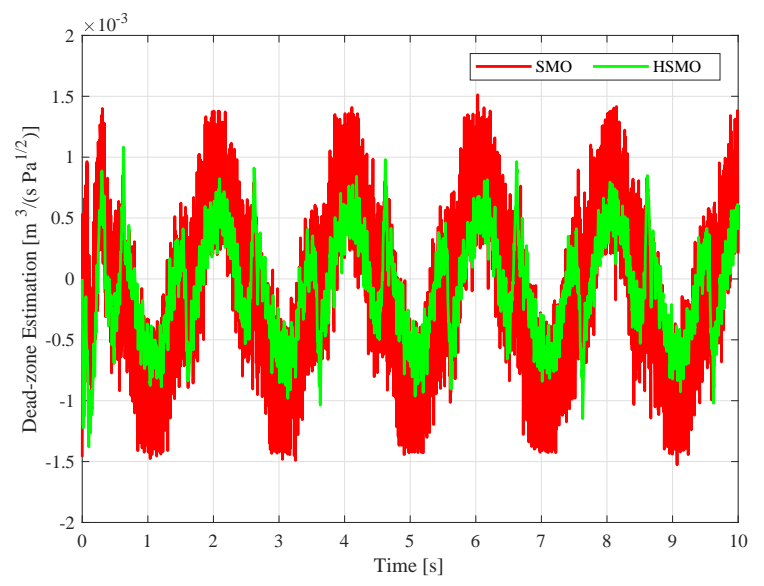

(b) Valve dead-zone estimation provided by the SMO and the HSMO.

Figure 10: Pressure inside the piston chamber and valve dead-zone during the experiment (black) and estimated by the EKF (blue), by the SMO (red) and by the HSMO (green).

Table 9: RMS value of the state estimation errors obtained during the experiment.

\begin{tabular}{|l|c|c|c|c|c|}
\hline & $y$ & $\dot{y}$ & $P_{L}$ & $v_{e}$ & $\dot{v}_{e}$ \\
\hline EKF & $1.8376 \mathrm{e}-5$ & $7.0581 \mathrm{e}-4$ & $7.3312 \mathrm{e}+3$ & $7.2157 \mathrm{e}-4$ & 0.0583 \\
\hline SMO & $5.6111 \mathrm{e}-8$ & $1.2418 \mathrm{e}-4$ & 129.56 & $2.3022 \mathrm{e}-4$ & 0.0121 \\
\hline HSMO & $2.2120 \mathrm{e}-7$ & $6.2318 \mathrm{e}-5$ & 63.753 & $1.6682 \mathrm{e}-4$ & 0.0136 \\
\hline
\end{tabular}

The results of the experimental test are shown in Fig. 9, 10 and 11. In particular, Fig. 9 reports the piston position, velocity and the estimated friction. Figure 9 (a) shows the piston position and the position estimated by the three observers together with the comparison between the estimation errors. In this case, it is possible to see from the plot of the piston position estimation error reported in the bottom plot of Fig. 9(a) that the EKF shows a large error with respect to the SMO and the HSMO. On the other hand, the SMO and the HSMO perform similarly and, if compared with the previous simulation in noisy condition, the SMO performs very well since the measurement noise is smaller than the ones evaluated in simulation.

Figure 9(b) reports, in the top plot, the piston velocity and the one estimated by the EKF, the SMO and the HSMO while the bottom plot reports the comparison between the estimation error of the EKF, the SMO and the HSMO, to highlight the larger error of both the EKF and the SMO with respect to the HSMO. From the piston velocity estimation error reported in the bottom plot of Fig. 9(b) it can be seen that the HSMO provides a better estimation of the state with respect to the EKF and the SMO.

Finally, Fig. 9(c) shows the friction acting on the piston estimated by the SMO and the HSMO (the EKF does not provide any disturbance estimation). From this plot it can be seen that the the SMO does not provide a meaningful friction estimation, while the HSMO estimate a friction even larger than the one adopted during the simulation.

The pressure in the piston chamber and the effect of the valve dead-zone are reported in Fig. 10. In particular, Fig. 10(a) reports the piston pressure and the one estimated by the EKF, the SMO and the HSMO together with the comparison between their estimation errors, to highlight the very large error of the EKF with respect to the SMO and the HSMO. It is possible to see from the plot of the chamber pressure estimation error reported in the bottom plot of Fig. 10(a) that the HSMO performs better than the other observers, even if the reduced measurement noise with respect to the previous simulations allows to the SMO to provide a reliable estimation.

For what related to the valve dead-zone, Fig. 10(b) reports the estimation provided by the SMO and the HSMO: by comparing this plot with the simulation results, it is clear in particular from the estimation provided by the HSMO that the valve dead-zone presents a more complex behavior with respect the simulations.

Figure 11 reports the valve data. In particular, Fig. 11(a) and 11(b) show, in the top plots, the valve position and velocity and the ones estimated by the EKF, the SMO and the HSMO, while the bottom plots report the comparison between the estimation errors of the three observers. From these plots, it possible to see that in this case the three observers performs in a similar way, with the HSMO estimation error apparently less affected by the respective 

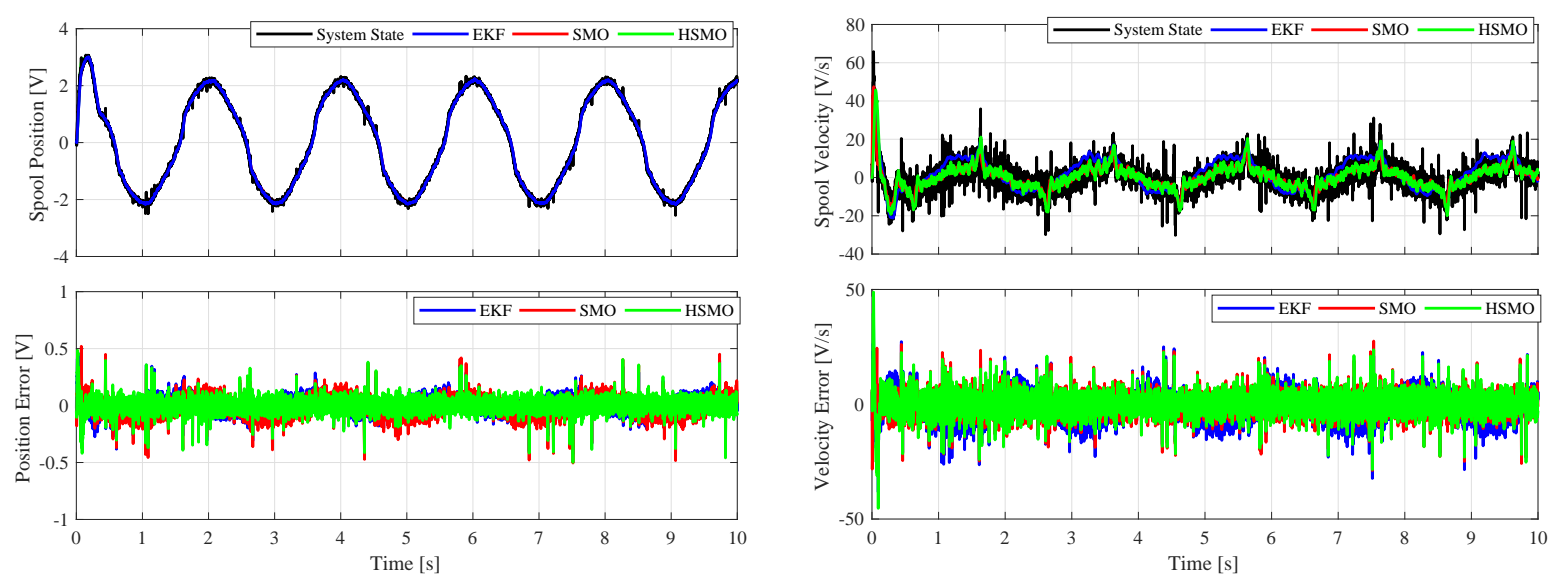

(a) Spool position. Top plot: valve position and estimation by the EKF, the SMO and the HSMO. Bottom plot: comparison between the estimation errors of the three observers.

(b) Spool velocity. Top plot: valve velocity and estimation by the EKF, the SMO and the HSMO. Bottom plot: comparison between the estimation errors of the three observers.

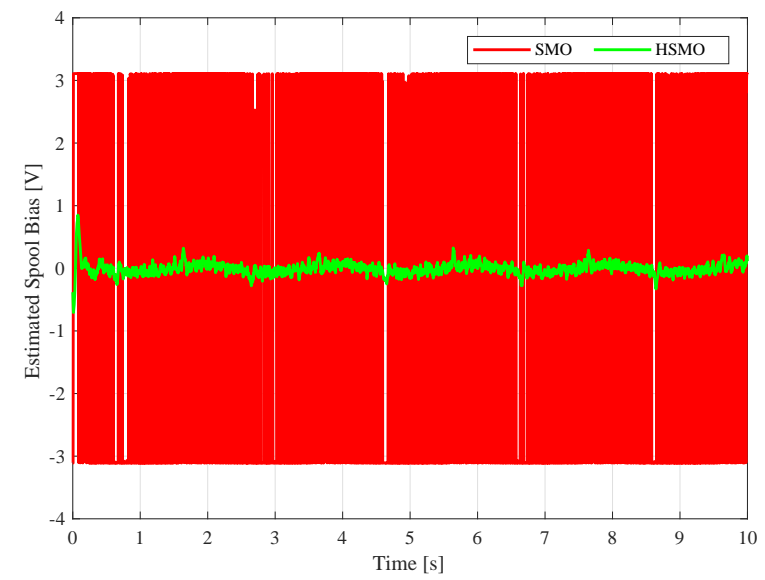

(c) Valve input bias estimated by the SMO and the HSMO.

Figure 11: Valve position, velocity and input bias during the experiment (black) and estimated by the EKF (blue), by the SMO (red) and by the HSMO (green).

output variations but from the measurement noise only.

Finally, Fig. 11(c) shows the valve input bias estimated by the SMO and the HSMO (the EKF does not provide any disturbance estimation). Also in this case, the HSMO shows the advantage of providing a reliable disturbance estimation.

The performance of the designed observers in terms RMS value of the state estimation errors during the experimental test are reported in Tab. 9. These data show that both the SMO and the HSMO provide good performance in terms of state estimation, but the HSMO significantly outperforms the SMO in terms of meaningful disturbance estimation.

\section{Conclusions}

In this paper, the evaluation of different robust observation methods applied to a hydraulic actuation system is reported. A SMO and a HSMO are compared with an EKF considered as a standard approach for the state observation in case of noisy measurements and uncertain systems.

The comparison between the observer responses has been executed in simulation first assuming perfect output knowledge, to evaluated the tuning of the observers and the general properties of the different methods, in particular with respect to the state observation error and the disturbance estimation. Secondly, simulations with noisy outputs have been carried out to verify the sensitivity of the observers with respect to noise.

Finally, an experimental test reproducing the same working conditions evaluated in simulation have been presented to highlight the characteristics of the different approaches in real conditions. 
From this analysis, it can be summarized that both the SMO and the HSMO show better performance with respect to the EKF, and that the HSMO presents in general a more reliable behavior even in presence of noisy outputs and large external disturbance. A noticeable additional feature of the HSMO is the ability of providing a reliable estimation of the disturbance acting on the system, which can be exploited for diagnosis purpose and fault detection. Moreover, the design procedure of the HSMO is simpler and with a smaller set of parameters to tune with respect to the SMO.

Future work will be devoted to investigate the possibility of reducing the number of sensors in the system and to evaluate the system behavior in different loading conditions and during specific working cycles.

\section{References}

[1] R. Isermann, Supervision, fault-detection and fault-diagnosis methods an introduction, Control Engineering Practice 5 (5) (1997) 639-652.

[2] P. M. Frank, On-line fault detection in uncertain nonlinear systems using diagnostic observers: a survey, International Journal of Systems Science 25 (12) (1994) 2129-2154.

[3] F. Abry, X. Brun, M. Di Loreto, S. Sesmat, . Bideaux, Piston position estimation for an electro-pneumatic actuator at standstill, Control Engineering Practice 41 (2015) 176-185.

[4] N. Niksefat, N. Sepehri, A qft fault-tolerant control for electrohydraulic positioning systems, IEEE Transactions on Control Systems Technology 10 (4) (2002) 626-632.

[5] X.-b. Fu, B. Liu, Y.-c. Zhang, L.-n. Lian, Fault diagnosis of hydraulic system in large forging hydraulic press, Measurement 49 (2014) 390-396.

[6] A. Maddahi, W. Kinsner, N. Sepehri, Internal leakage detection in electrohydrostatic actuators using multiscale analysis of experimental data, IEEE Transactions on Instrumentation and Measurement 65 (12) (2016) 27342747.

[7] H.-Z. Tan, N. Sepehri, Parametric fault diagnosis for electrohydraulic cylinder drive units, IEEE Transactions on Industrial Electronics 49 (1) (2002) 96-106.

[8] C. C. DeBoer, B. Yao, Velocity control of hydraulic cylinders with only pressure feedback, in: ASME International Mechanical Engineers Congress and Exposition, 2001.

[9] T. Virtalo, Nonlinear model of analog valve, in: Proc. of the 5th Scandinavian International Conference on Fluid Power, 1997, pp. 199-214.

[10] D. Yu, Fault diagnosis for a hydraulic drive system using a parameter-estimation method, Control Engineering Practice 5 (9) (1997) 1283-1291.

[11] D. N. Shields, S. Daley, Application of a robust nonlinear fault detection observer to a hydraulic system, in: 1997 European Control Conference (ECC), 1997, pp. 289-294.

[12] H. Hammouri, P. Kabore, S. Othman, J. Biston, Failure diagnosis and nonlinear observer. application to a hydraulic process, Journal of the Franklin Institute 339 (4) (2002) 455-478.

[13] L. An, N. Sepehri, Hydraulic actuator circuit fault detection using extended kalman filter, in: Proceedings of the 2003 American Control Conference, 2003., Vol. 5, 2003, pp. 4261-4266.

[14] M. Khoshzaban, F. Sassani, P. D. Lawrence, Online state and parameter estimation of an electrohydraulic valve for intelligent monitoring, in: Proceedings of IEEE/ASME International Conference on Advanced Intelligent Mechatronics, 1997.

[15] P. Halder, A novel approach for detection and diagnosis of process and sensor faults in electro-hydraulic actuators, International Journal of Engineering Research and Development 6 (7) (2013) 15-22.

[16] M. Sepasi, F. Sassani, On-line fault diagnosis of hydraulic systems using unscented kalman filter, International Journal of Control, Automation and Systems 8 (1) (2010) 149-156.

[17] C. Abagnale, F. Aggogeri, A. Borboni, S. Strano, M. Terzo, Dead-zone effect on the performance of state estimators for hydraulic actuators, Meccanica 52 (9) (2017) 2189-2199. 
[18] R. Rajamani, J. K. Hedrick, Adaptive observers for active automotive suspensions: theory and experiment, IEEE Transactions on Control Systems Technology 3 (1) (1995) 86-93.

[19] P. Garimella, B. Yao, Nonlinear adaptive robust observer design for a class of nonlinear systems, in: Proceedings of the American Control Conference, Vol. 5, 2003, pp. 4391-4396.

[20] P. Garimella, B. Yao, Nonlinear adaptive robust observer for velocity estimation of hydraulic cylinders using pressure measurement only, in: ASME International Mechanical Engineering Congress and Exposition, Dynamic Systems and Control, New Orleans, Louisiana, USA, 2002.

[21] A. Mohanty, S. Gayaka, B. Yao, An adaptive robust observer for velocity estimation in an electro-hydraulic system, International Journal of Adaptive Control and Signal Processing 26 (12) (2012) 1076-1089.

[22] A. Levant, Sliding order and sliding accuracy in sliding mode control, International Journal of Control 58 (6) (1993) 1247-1263.

[23] F. Zhu, J. Yang, Fault detection and isolation design for uncertain nonlinear systems based on full-order, reduced-order and high-order high-gain sliding-mode observers, International Journal of Control 86 (10) (2013) $1800-1812$.

[24] H. Ros, J. Davila, L. Fridman, High-order sliding mode observers for nonlinear autonomous switched systems with unknown inputs, Journal of the Franklin Institute 349 (10) (2012) 2975-3002.

[25] J. J. Rath, K. C. Veluvolu, M. Defoort, Y. C. Soh, Higher-order sliding mode observer for estimation of tyre friction in ground vehicles, IET Control Theory Applications 8 (6) (2014) 399-408.

[26] L. Fridman, Y. Shtessel, C. Edwards, X.-G. Yan, Higher-order sliding-mode observer for state estimation and input reconstruction in nonlinear systems, International Journal of Robust and Nonlinear Control 18 (4-5) (2008) 399-412.

[27] T. Floquet, J. P. Barbot, Super twisting algorithm-based step-by-step sliding mode observers for nonlinear systems with unknown inputs, International Journal of Systems Science 38 (10) (2007) 803-815.

[28] Y. Zhou, Y. Soh, J. Shen, High-gain observer with higher order sliding mode for state and unknown disturbance estimations, International Journal of Robust and Nonlinear Control 24 (15) (2014) 2136-2151.

[29] A. Bonchis, P. I. Corke, D. C. Rye, A pressure-based, velocity independent, friction model for asymmetric hydraulic cylinders, in: Proceedings 1999 IEEE International Conference on Robotics and Automation, Vol. 3, 1999, pp. 1746-1751.

[30] H. Merritt, Hydraulic Control Systems, Wiley, 1967.

[31] S. Strano, M. Terzo, A multi-purpose seismic test rig control via a sliding mode approach, Structural Control and Health Monitoring 21 (8) (2014) 1193-1207.

[32] L. Mrton, S. Fodor, N. Sepehri, A practical method for friction identification in hydraulic actuators, Mechatronics 21 (1) (2011) 350-356.

[33] Y. Xiong, M. Saif, Sliding-mode observer for uncertain systems. II. Nonlinear systems case, in: Proc. IEEE Conf. on Decision and Control, Vol. 1, 2000, pp. 322-327.

[34] A. Levant, Higher-order sliding modes, differentiation and output-feedback control, International Journal of Control 76 (9-10) (2003) 924-941. 